1999-06-01

\title{
Creatine Supplementation Differentially Affects Maximal Isometric Strength and Time to Fatigue in Large and Small Muscle Groups
}

William J. Vincent

bill_vincent@byu.edu

Steven F. Loy

Richard L. Urbanski

Ben B. Yaspelkis

Follow this and additional works at: https://scholarsarchive.byu.edu/facpub

Part of the Exercise Science Commons

\section{Original Publication Citation}

Creatine Supplementation Differentially Affects Maximal Isometric Strength and Time to Fatigue in Large and Small Muscle Groups. International Journal of Sports Nutrition. Vol. 9, \#2, pp. 136 145, June 1999. (R. L. Urbanski, S. F. Loy, W. J. Vincent, B. B. Yaspelkis).

\section{BYU ScholarsArchive Citation}

Vincent, William J.; Loy, Steven F.; Urbanski, Richard L.; and Yaspelkis, Ben B., "Creatine Supplementation Differentially Affects Maximal Isometric Strength and Time to Fatigue in Large and Small Muscle Groups" (1999). Faculty Publications. 617.

https://scholarsarchive.byu.edu/facpub/617

This Peer-Reviewed Article is brought to you for free and open access by BYU ScholarsArchive. It has been accepted for inclusion in Faculty Publications by an authorized administrator of BYU ScholarsArchive. For more information, please contact ellen_amatangelo@byu.edu. 


\title{
Creatine Supplementation Differentially Affects Maximal Isometric Strength and Time to Fatigue in Large and Small Muscle Groups
}

\author{
Richard L. Urbanski, Steven F. Loy, William J. Vincent, \\ and Ben B. Yaspelkis III
}

\begin{abstract}
Ten physically active, untrained, college-aged males ( $26.4 \pm 5.8$ years old) received creatine ( $\mathrm{CR}, 5 \mathrm{~g}$ creatine monohydrate $+3 \mathrm{~g}$ dextrose) and placebo (PLA, $7 \mathrm{~g}$ dextrose) supplementation four times per day for 5 days in a doubleblind, randomized, balanced, crossover design. Performance was assessed during maximal and three repeated submaximal bouts of isometric knee extension and handgrip exercise. CR supplementation significantly increased $(p<.05)$ maximal isometric strength during knee extension but not during handgrip exercise. CR supplementation increased time to fatigue during each of the three bouts of submaximal knee extension and handgrip exercise when compared to the PLA trials. These findings suggest that CR supplementation can increase maximal strength and time to fatigue during isometric exercise. However, the improvements in maximal isometric strength following CR supplementation appear to be restricted to movements performed with a large muscle mass.
\end{abstract}

Key Words: ergogenic aid, phosphocreatine, sport nutritional performance supplement

Although the specific physiological mechanisms that contribute to fatigue during multiple bouts of short-duration, high-intensity exercise are not fully understood, it appears that depletion of skeletal muscle phosphocreatine ( $\mathrm{PCr}$ ) is a significant causative factor. Hargreaves et al. (11) recently reported that exercise performance during high-intensity intermittent exercise is limited by $\mathrm{PCr}$ availability. Furthermore, Bogdanis et al.(3) showed that the rate of $\mathrm{PCr}$ resynthesis contribute to recovery of power output during repeated bouts of sprint exercise. Thus, if skeletal muscle $\mathrm{PCr}$ stores could be elevated prior to exercise, time to fatigue could be delayed and recovery could be enhanced.

A number of investigators have shown that oral creatine supplementation can increase resting $\mathrm{PCr}$ stores as well as increase the rate of $\mathrm{PCr}$ resynthesis following exhaustive exercise $(9,10,12,15)$. Harris et al. (12) demonstrated that an oral supplementation regime of $20 \mathrm{~g} /$ day of creatine monohydrate for 3-7 days elevated

The authors are with the Exercise Biochemistry Laboratory, Department of Kinesiology, California State University, Northridge, 18111 Nordhoff St., Northridge, CA 913308287. Direct correspondence to B.B. Yaspelkis III. 
total creatine $(\mathrm{TCr}$ ) levels in skeletal muscle by $\sim 20 \%$. $\mathrm{PCr}$ plays a major role in energy metabolism by providing a phosphate group to rephosphorylate adenosine diphosphate (ADP) to adenosine triphosphate (ATP). Thus creatine supplementation may enhance performance during short-duration, high-intensity events by enhancing the rate of ATP synthesis during muscle contraction and may facilitate recovery by enhancing the rate of $\mathrm{PCr}$ resynthesis.

A number of investigators have demonstrated a positive effect of creatine supplementation on exercise performance $(1,2,6,10,12,17,28,29)$, providing reasonable support for the ergogenic potential of creatine. However, not all investigators have shown creatine supplementation to enhance exercise performance $(4,5$, $7,20-22,25)$. As indicated by Snow et al. (25), a potential point of conflict may be that many of these investigators used cross-sectional or ordered treatment experimental designs as opposed to crossover designs due to the 4-week period needed to wash out creatine (15). It is also possible that the differences observed between investigations arise from how exercise performance was evaluated. Creatine supplementation does not appear to enhance a single bout of exercise $(1,4,5,7,10,20-22$, 25 ) but rather appears to exert its primary ergogenic effect during the later bouts of intermittent exercise by increasing the body's ability to resynthesize $\mathrm{PCr}$ between exercise bouts $(1,2,10)$.

The effects of creatine supplementation on exercise performance have been investigated primarily using dynamic, isotonic contractions. However, to more fully evaluate the mechanism by which creatine improves performance, some investigators have used isometric contractions $(17,19,27)$. Isometric contractions that are performed at greater than $67 \%$ of the maximal isometric strength of the muscle occlude blood flow and bring about anoxia within the first few seconds of contraction (13). When blood flow to a working muscle is reduced, the contractile force declines due to a reduction in ATP availability and a decreased ability to resynthesize $\mathrm{PCr}$ (14). If force production and time to fatigue are to be improved during isometric contractions, then the ATP-PCr system must be enhanced to support this activity. Therefore, the primary purpose of this investigation was to determine if maximal isometric strength and time to fatigue during repeated submaximal isometric contractions were improved following creatine supplementation using a double-blind, crossover experimental design.

$\mathrm{TCr}$ levels in skeletal muscle typically range from 115 to $140 \mathrm{mmol} / \mathrm{kg}$ dry muscle (12) and can be increased by $\sim 20-40 \mathrm{mmol} / \mathrm{kg}$ dry muscle in humans following 5 days of creatine supplementation at $20 \mathrm{~g} /$ day $(8,9,12,15)$. However, it is unknown if creatine supplementation differentially affects exercise performance depending on the quantity of the muscle mass recruited to complete the activity. Therefore, a secondary purpose of this investigation was to characterize the roles of active muscle mass in determining the effect of creatine supplementation on exercise performance during isometric knee extension and handgrip exercises.

\section{Methods}

\section{Subjects}

Ten healthy male subjects who were $26.4 \pm 5.8$ years of age and weighed $77.1 \pm 12.6$ $\mathrm{kg}$ were recruited to participate in the study. All participants reported being physically active but were not highly trained in the movements tested. Subjects were fully 
informed of the purpose of the study and of any potential risks of participating. All participants completed a health history questionnaire and informed consent before commencing the study. Formal approval to conduct this study was gained from the Standing Advisory Committee for the Protection of Human Subjects, California State University, Northridge.

\section{Experimental Design}

A double-blind, counterbalanced, crossover design was used such that each subject completed a creatine (CR) and a placebo (PLA) trial. Each treatment trial involved a presupplement exercise session, a 5-day supplementation period, and a postsupplement exercise session. The CR and PLA trials were separated by no less than 35 days to allow elevated skeletal muscle creatine stores to return to basal levels (15).

During the presupplement exercise session, all subjects reported to the laboratory where they received specific instructions for the supplementation period, were familiarized with the test equipment, and practiced the exercise protocols. Subjects were asked to maintain their current activity level during the 5-day supplementation period and to refrain from any high-intensity strength training or isometric exercises that would simulate those performed in the study. Subjects recorded their dietary intakes during the initial 5-day treatment period and were required to duplicate their dietary intakes during the second 5-day treatment period.

\section{Supplementation Protocol}

Following the presupplementation exercise session, subjects were given either a PLA or CR supplement package. Each supplement package contained 20 individual PLA or CR dosage containers with premeasured, powdered supplement mixes. The PLA dose consisted of $7 \mathrm{~g}$ of a commercially available carbohydrate powder, and the $\mathrm{CR}$ dose consisted of $5 \mathrm{~g}$ of creatine monohydrate plus $3 \mathrm{~g}$ of the carbohydrate powder. Subjects were instructed to dissolve the contents of one individual dosage container in $8 \mathrm{oz}$ of tap water and consume the liquid; they were told to repeat this four times per day, $3-4 \mathrm{hr}$ apart, for 5 days. This protocol resulted in ingestion of 20 $\mathrm{g} /$ day of creatine monohydrate for 5 days during the $\mathrm{CR}$ trial, an amount that significantly elevates skeletal muscle creatine stores $(8,9,12,15)$.

Green et al. reported that providing creatine in conjunction with a carbohydrate source augments creatine accumulation in skeletal muscle (8). However, the amount of carbohydrate used by Green et al. (8) was substantially greater than that provided in the present investigation. Nevertheless, CR was provided with the carbohydrate powder such that the treatments would appear similar in taste, color, and texture. Following the investigation, subjects were unable to identify with consistency the treatment order to which they were subjected, suggesting that they were properly blinded to the treatments throughout the experimental period.

\section{Exercise Protocol}

Following the 5-day supplementation period, the subjects reported to the laboratory to perform four separate isometric exercises that utilized a large muscle mass, knee extension (KE), and a small muscle mass, handgrip (HG). KE exercise was performed on a Biodex Test Station (Biodex Medical Systems Inc., Shirley, NY). 
Handgrip exercise was performed with a hand dynamometer (Jamar 5031J1, Jackson, MI). The four exercises performed were maximal isometric knee extension (MaxKE), submaximal isometric knee extension (SKE), maximal isometric handgrip (MaxHG), and submaximal isometric handgrip (SHG). A contraction intensity of $67 \%$ maximal contraction was chosen for the submaximal isometric exercises because it has been demonstrated that, at this intensity, blood flow to the working muscles is occluded and the muscle becomes anoxic within the first few seconds of contraction (13).

Testing was performed on the subject's dominant extremity. For KE, subjects assumed a fixed seated position on the Biodex chair in $90^{\circ}$ of hip flexion and $90^{\circ}$ of knee flexion. The torso and thigh were fastened to the chair using the securing straps provided on the Biodex test station. For HG, the subjects were in a seated position with the forearm supported in a relaxed position maintaining $90^{\circ}$ of elbow flexion and with the shoulder in a neutral position.

The order of exercise performance was MaxKE, MaxHG, SKE, and SHG. Subjects were given a 5-min rest period between exercises. MaxKE and MaxHG were determined as the best of three maximal isometric contractions that were performed $60 \mathrm{~s}$ apart. For SKE, subjects were presented a graphic display of the amount of isometric torque being produced during contraction. This display included a line that represented $67 \%$ of the subjects' maximal isometric strength. Subjects were instructed to initiate contraction of the knee extensors, bring their isometric torque production to $67 \%$ MaxKE as quickly as possible, and then maintain contraction intensity until fatigue. When isometric torque could no longer be sustained, the time of contraction was recorded and the subjects were allowed to rest for $60 \mathrm{~s}$. This sustained isometric contraction protocol was performed three times. The SHG protocol was similar to the SKE except that the subjects' visual representation of the $67 \%$ contraction intensity was provided with the peak hold needle of the dynamometer positioned at $67 \%$ MaxHG. Constant verbal encouragement to maintain force production was given to the subjects during each bout of SHG and SKE.

\section{Statistical Analysis}

We analyzed maximal isometric performance for KE and HG using Student's $t$ test to compare PLA and CR treatments. Times to fatigue during the submaximal isometric exercise bouts were analyzed using a two-way analysis of variance with repeated measures to compare CR and PLA treatments. When a significant $F$ ratio was obtained, we employed Tukey's post hoc test to identify differences among the means. The level of statistical significance was set at $p<.05$.

\section{Results}

CR supplementation significantly increased MaxKE torque by $\sim 4.2 \%$ when compared to the PLA treatment (Table 1). CR supplementation also increased time to fatigue during submaximal isometric knee extension (Figure 1). Time to fatigue was increased by $11 \%, 18 \%$, and $22 \%$, respectively, in CR SKE Bouts 1,2 , and 3 when compared to the PLA trials.

Maximal isometric handgrip strength following CR was not significantly different than PLA results (Table 1). However, time to fatigue during SHG was significantly increased following CR supplementation when compared to the PLA 
treatment (Figure 2). CR supplementation increased time to fatigue by $16 \%$, $21 \%$, and $18 \%$, respectively, during CR SHG Bouts 1,2 , and 3 when compared to the PLA trials.

Prior to supplementation, subjects' mean body weights were $77.2 \pm 4.0 \mathrm{~kg}$ and $77.1 \pm 4.1 \mathrm{~kg}$, respectively, for the PLA and CR treatments. Mean body weight following PLA treatment was $77.2 \pm 4.1 \mathrm{~kg}$, whereas body weight was $77.9 \pm 4.1 \mathrm{~kg}$ following CR supplementation. However, the increase in body weight following CR supplementation was not statistically significant $(p=.91)$.

Table 1 Maximal Isometric Performance During Knee Extension and Handgrip Exercise

\begin{tabular}{lcccr}
\hline & \multicolumn{2}{c}{ Placebo } & \multicolumn{2}{c}{ Creatine } \\
& $M$ & $S E$ & $M$ & $S E$ \\
\hline Knee extension $(\mathrm{kg} \cdot \mathrm{m})$ & 28.96 & 1.5 & $30.19^{*}$ & 1.6 \\
Handgrip $(\mathrm{kg})$ & 57.1 & 2.8 & 57.3 & 2.6 \\
& & & &
\end{tabular}

Note. Subjects consumed a placebo or $20 \mathrm{~g} /$ day creatine monohydrate for 5 days prior to testing.

*Significantly different from placebo $(p<.05)$.

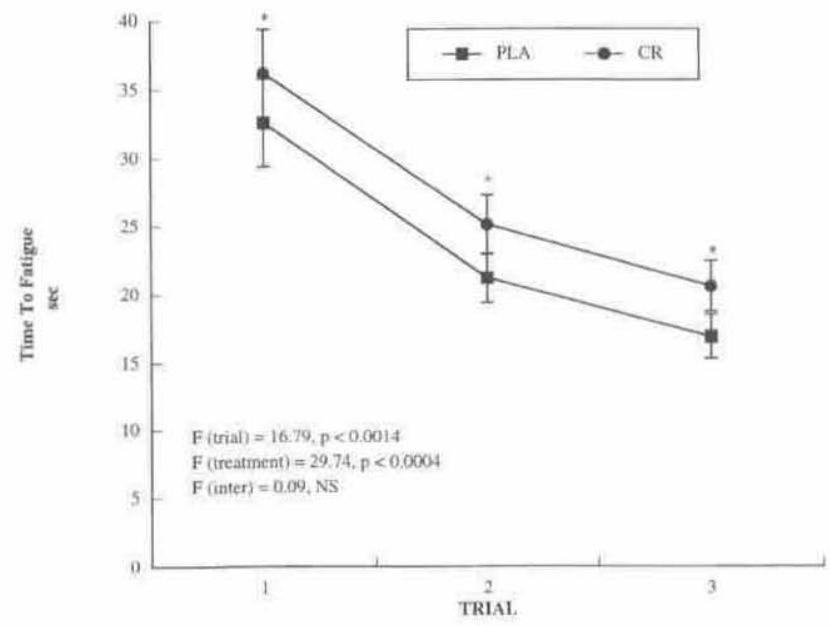

Figure 1 - Time to fatigue during submaximal isometric knee extension. Each trial was separated by $60 \mathrm{~s}$. PLA = placebo; CR = creatine. Values are means $\pm S E$.

*Significantly different from PLA $(p<.01)$. 


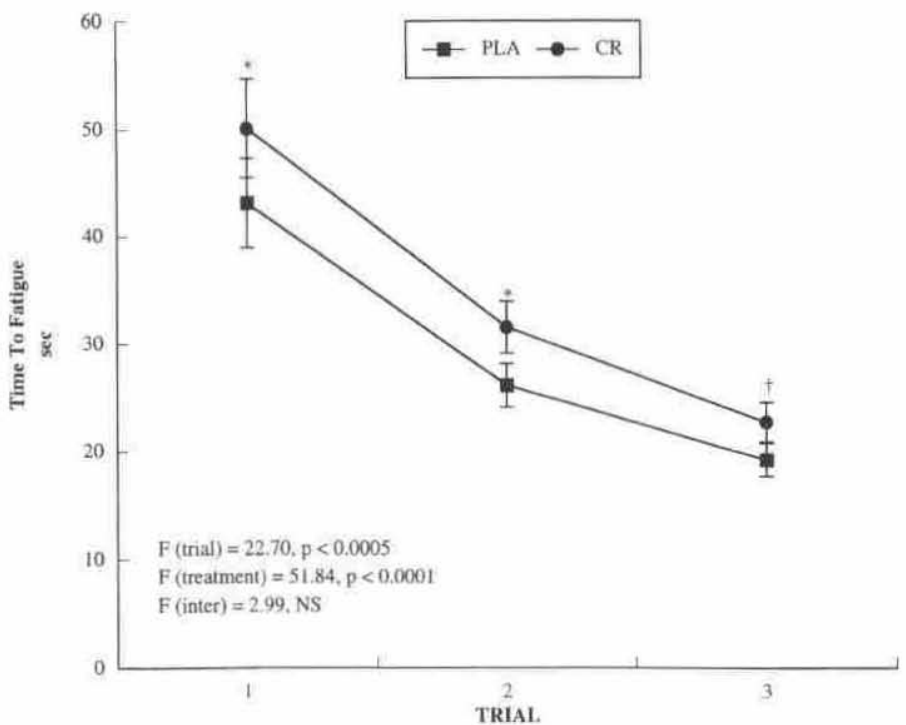

Figure 2 - Time to fatigue during submaximal isometric handgrip exercise. Each trial was separated by $60 \mathrm{~s}$. PLA = placebo; $C R=$ creatine. Values are means $\pm S E$.

$*$ Significantly different from PLA $(p<.01)$.

$\dagger$ Significantly different from PLA $(p<.05)$.

\section{Discussion}

The effect of creatine supplementation on enhancing performance during dynamic exercise has been extensively studied (1, 2, 4-7, 10, 12, 20-22, 25, 28, 29); however, little information has been presented to suggest whether creatine supplementation can improve isometric exercise performance $(17,19,27)$. Isometric contractions performed at more than $67 \%$ of the maximal isometric force of the muscle occlude blood flow (13), reduce ATP availability, and decrease the rate of $\mathrm{PCr}$ resynthesis (14). Such an ischemic effect can impair the contraction process and reduce or completely halt force production. If isometric performance is to be improved, ATP availability and $\mathrm{PCr}$ resynthesis must be enhanced.

In the present investigation, we found that 5 days of CR supplementation (20 $\mathrm{g} /$ day) significantly increased time to fatigue during repeated bouts of submaximal isometric handgrip and knee extension exercise. This observation is in agreement with findings of Greenhaff et al. (10), who reported that creatine supplementation increased peak torque production during repeated bouts of isokinetic knee extension. Five days of creatine supplementation at $20 \mathrm{~g} /$ day will increase $\mathrm{TCr}$ levels in human skeletal muscle by $\sim 20-40 \mathrm{mmol} / \mathrm{kg}$ dry muscle but will not elevate the skeletal muscle ATP concentration $(8,9,12)$. Thus, the improvements in time to fatigue during the repeated bouts of SHG and SKE following CR treatment likely resulted from an elevated skeletal muscle PCr concentration enhancing ATP maintenance during contraction as well as facilitating $\mathrm{PCr}$ resynthesis between the exercise trials. 
A surprising observation in the present investigation was that time to fatigue was significantly increased in the initial bouts of both SHG and SKE. A substantial body of evidence demonstrates that creatine supplementation will enhance performance only during repeated bouts of high-intensity, dynamic exercise $(1,2,6,10$, 12,29). Creatine supplementation does not appear to improve performance during a single bout of dynamic exercise $(1,4,5,7,10,20-22,25)$. However, our observation that creatine supplementation increased time to fatigue in the initial bout of submaximal exercise can likely be attributed to the mode of contraction used in the investigation. Our subjects performed isometric contractions at an intensity that would have significantly reduced ATP availability to the working musculature (13). Consequently, a greater reliance would likely have been placed on the anaerobic energy production systems to sustain force production during this type of activity than would occur during a single bout of dynamic exercise. Thus, the increased time to fatigue during the initial bout of SHG and SKE appears to have resulted from the CR treatment enhancing ATP maintenance during the isometric contraction.

CR supplementation also differentially affected improvements in time to fatigue across the repeated trials of SHG and SKE. Time to fatigue was increased $\sim 3.7 \mathrm{~s}$ for all three trials of SKE during the CR treatment when compared to the PLA treatment. In contrast, the difference in time to fatigue during SHG decreased $50 \%$ from Trial 1 to Trial 3 when the CR and PLA trials are compared. This finding suggests that the ability of creatine to enhance performance by increasing time to fatigue during repeated bouts of exercise might be greater in activities involving larger muscle groups.

In addition, we observed that the CR treatment, independent of exercise training, increased maximal isometric performance during MaxKE by $\sim 4.2 \%$ but did not increase MaxHG. To our knowledge, this is the first investigation to report that creatine supplementation can increase maximal isometric strength in some, but not all, muscle groups within the same subjects. Of particular interest was that the difference in isometric strength performance following creatine supplementation appeared to be related to the amount of muscle mass involved during the contraction effort. However, the physiological mechanism that accounted for this difference in maximal isometric performance between knee extension and handgrip exercise following creatine supplementation warrants further investigation. We used subjects not specifically trained in the movements tested; if the subjects had been highly trained, the outcomes of this investigation might have been different.

Maximal isometric strength was recorded as the peak force that was produced within $2 \mathrm{~s}$ following the initiation of muscle contraction. During the initial seconds of a maximal contraction, the energy for the contraction is provided by the ATP stored in the skeletal muscle (26). However, creatine supplementation does not increase skeletal muscle ATP levels $(8,9,12)$. Rather, creatine supplementation enhances exercise performance by increasing skeletal muscle $\mathrm{PCr}$ stores, which increases ATP production in the ATP-PCr system. During a maximal isometric contraction that is completed within $2 \mathrm{~s}$, PCr stores are not rate limiting and contribute minimally to ATP production. Therefore, mechanisms other than elevations in skeletal muscle PCr stores must account for the increased MaxKE performance following CR treatment.

It is generally accepted that increases in maximal muscular strength are associated with an increase in the cross-sectional area of the muscle. Although body weight gain following creatine supplementation has been attributed to water retention 
(15), some investigators have speculated that increases in fat-free mass are due to muscle protein synthesis $(6,28)$. Ingwall $(16)$ demonstrated that creatine can stimulate myosin synthesis in incubated muscle cells, and Sipilä et al. (24) reported that low doses of creatine supplementation ( $1 \mathrm{~g} /$ day) increased Type II muscle fiber diameter and muscular strength in gyrate atrophy patients. Conversely, the administration of creatine analogues has been shown to retard muscle growth and promote muscle weakness in animals $(18,23)$. Although 5 days is a short time to expect creatine supplementation to significantly increase muscle protein accretion, in the absence of alternative explanations it might be speculated that cross-sectional area or possibly a remodeling of muscle fibers may account for the increased isometric performance during MaxKE. It remains to be determined, however, if creatine supplementation increases skeletal muscle protein synthesis in humans.

We found that $20 \mathrm{~g} /$ day of creatine for 5 days significantly increased maximal isometric strength during knee extension but not during handgrip exercise. This observation suggests that improvements in maximal isometric strength following creatine supplementation are restricted to contractions performed with a large muscle mass. In contrast, creatine supplementation increased time to fatigue during each of three repeated bouts of submaximal isometric handgrip and knee extension exercise.

\section{References}

1. Balsom, P.D., B. Ekblom, K. Söderlund, B. Sjodin, and E. Hultman. Creatine supplementation and dynamic high-intensity intermittent exercise. Scand. J. Med. Sci. Sports 3:143-149, 1993.

2. Balsom, P.D., K. Söderlund, B. Sjodin, and B. Ekblom. Skeletal muscle metabolism during short duration high-intensity exercise: Influence of creatine supplementation. Acta Physiol. Scand. 154:303-310, 1995.

3. Bogdanis, G.C., M.E. Nevill, L.H. Boobis, H.K. Lakomy, and A.M. Nevill. Recovery of power output and muscle metabolites following $30 \mathrm{~s}$ of maximal sprint cycling in man. $J$. Physiol. 482:467-480, 1995.

4. Burke, L.M., D.B. Pyne, and R.D. Telford. Effects of oral creatine supplementation on single-effort sprint performance in elite swimmers. Int. J. Sport Nutr. 6:222-233, 1996.

5. Cooke, W.H.,P.W. Grandjean, and W.S. Barnes. Effect of oral creatine supplementation on power output and fatigue during bicycle ergometry. J. Appl. Physiol. 78:670-673, 1995.

6. Earnest, C.P., P.G. Snell, R. Rodriguez, and A.L. Almada. The effect of creatine monohydrate ingestion on anaerobic power indices, muscular strength and body composition. Acta Physiol. Scand. 153:207-209, 1995.

7. Febbraio, M.A., T.R. Flanagan, R.J. Snow, S. Zhao, and M.F. Carey. The effect of creatine supplementation on intramuscular $\mathrm{TCr}$, metabolism and performance during intermittent, supramaximal exercise in humans, Acta Physiol. Scand. 155:387-395, 1995.

8. Green, A.L., E. Hultman, I.A. Macdonald, D.A. Sewell, and P.L. Greenhaff. Carbohydrate ingestion augments skeletal muscle creatine accumulation during creatine supplementation in humans. Am. J. Physiol. 271:E821-E826, 1996.

9. Greenhaff, P.L., A. Casey, A.H. Short, R. Harris, K. Söderlund, and E. Hultman. Effect of oral creatine supplementation on skeletal muscle phosphocreatine resynthesis. Am.J. Physiol. 266:E725-E730, 1994.

10. Greenhaff, P.L., A. Casey, A.H. Short, R. Harris, K. Söderlund, and E. Hultman. Influence of oral creatine supplementation on muscle torque during repeated bouts of maximal voluntary exercise in man. Clin. Sci. 84:565-571, 1993. 
11. Hargreaves, M., M.J. McKenna, D.G. Jenkins, S.A. Warmington, J.L. Li, R.J. Snow, and M.A. Febbraio. Muscle metabolites and performance during high-intensity, intermittent exercise. J.Appl. Physiol. 84:1687-1691, 1998.

12. Harris, R.C., K. Söderlund, and E. Hultman. Elevation of creatine in resting and exercised muscle of normal subjects by creatine supplementation. Clin. Sci. 83: 367-374, 1992.

13. Henriksson, J., A. Katz, and K. Sahlin. Redox state changes in human skeletal muscle after isometric contractions. J. Physiol. 380:441-451, 1986.

14. Hogan, M.C., B.L. Gladden, B. Grassi, C.M. Stary, and M. Samaja. Bioenergetics of contracting skeletal muscle after partial reduction of blood flow.J. Appl. Physiol. 84:1882$1888,1998$.

15. Hultman, E.K., K. Söderlund, J.A. Timmons, G. Cederblad, and P.L. Greenhaff. Muscle creatine loading in man. J. Appl. Physiol. 81:232-237, 1996.

16. Ingwall, J.S. Creatine and the control of muscle specific protein syntheis in cardiac and skeletal muscle. Cir. Res. 38(Suppl. I):I-115-I-123, 1976.

17. Kurosawa, Y., H. Iwane, T. Hamoaka, T. Shimomitsu, T. Katsumura, T. Sako, M. Kuwamon, and N. Kimuta. Effects of oral creatine supplementation on high- and lowintensity grip exercise performance. Med. Sci. Sports Exerc. 29:S251, 1997.

18. Laskowski, M.B., R. Chevli, and C.D. Fitch. Biochemical and ultrastructural changes in skeletal muscle induced by a creatine antagonist. Metabolism 30:1080-1085, 1981.

19. Lemon, P., M. Boska, D. Bredle, M. Rogers, T.Ziegenfuss, and B. Newcomer. Effect of oral creatine supplementation on energetics during repeated maximal muscle contraction. Med. Sci. Sports Exerc. 27:S204, 1995.

20. Mujika, I., J.C. Chatard, L. Lacosote,F. Barale, and A. Geyssant. Creatine supplementation does not improve sprint performance in competitive swimmers. Med. Sci. Sports Exerc. 28:1435-1441, 1996.

21. Odland, L.M., J.D. MacDougall, M.A. Tarnopolsky, A. Elorriaga, and A. Brogmann. Effect of oral creatine supplementation on muscle [PCr] and short-term maximum power output. Med. Sci. Sports Exerc. 29:216-219, 1997.

22. Redondo, D.R., E.A. Dowling, B.L. Graham, A.L. Almada, and M.H. Williams. The effect of oral creatine monohydrate supplementation on running velocity. Int. J. Sport Nutr. 6:213-221, 1996.

23. Shields, R.P., C.K. Whitehair, R.E. Carrow, W.W. Heusner, and W.D.V. Huss. Skeletal muscle function and structure after depletion of creatine. Lab. Invest. 33:151-158, 1975.

24. Sipilä, I., J. Rapola, O. Simell, and A. Vamus. Supplementary creatine as a treatment for gyrate atrophy of the choroid and retina. N. Engl. J. Med. 304:867-870, 1981.

25. Snow, R.J., M.J. McKenna, S.E. Selig, J. Kemp, C.G. Stathis, and S. Zhao. Effect of creatine supplementation on sprint exercise performance and muscle metabolism. $J$. Appl. Physiol. 84:1667-1673, 1998.

26. Tullson, P.C., and R.L. Terjung. Adenine nucleotide metabolism in contracting skeletal muscle. In Exercise and Sport Science Reviews, J.O. Holloszy (Ed.). Baltimore: Williams \& Wilkins, 1991,pp. 507-537.

27. Vandenberghe, K., N. Gilles, M.V.Leemputte, P.V. Hecke, F. Vanstapel, and P. Hespel. Caffeine counteracts the ergogenic action of muscle creatine loading. J. Appl. Physiol. $80: 452-457,1996$.

28. Vandenberghe, K., M. Goris, P.V.Hecke, M.V.Leemputte, L. Vangerven, and P. Hespel. Long-term creatine intake is beneficial to muscle performance during resistance training. J. Appl. Physiol. 83:2055-2063, 1997. 
29. Volek, J.S., W.J. Kraemer, J.A. Bush, M. Boetes, T. Incledon, K.L. Clark, and J.M. Lynch. Creatine supplementation enhances muscular performance during high-intensity resistance exercise. J.Am. Diet Assoc. 97:765-770, 1997.

Manuscript received: July 20, 1998

Accepted for publication: October 9, 1998 